\title{
Strange stars in low-mass binary pulsar systems
}

\author{
J. E. Horvath \\ Instituto Astronômico e Geofísico, Universidade de São Paulo \\ Av. Miguel Stefano 4200 - São Paulo SP, 04301-904, Brazil \\ and \\ Steward Observatory, U. of Arizona \\ N. Cherry Av. 930, Tucson Arizona, USA
}

\begin{abstract}
Based on observational facts and a variety of theoretical arguments we discuss in this work the possibility that pulsars in Low-Mass Binary Pulsar systems could be strange stars rather than neutron stars. It is shown that, although subject to reasonable uncertainties, the consideration of the physics of the SQM core and thin normal crusts leads to the prediction of several observed features of the magnetic field history of these systems whitin this working hypothesis.
\end{abstract}

Pacs 97.04.40.Dg

Submitted to Int. Jour. Mod. Phys. D 


\section{INTRODUCTION}

Low-Mass Binary Pulsars (hereafter LMBP) are generally regarded as important to probe the effects of substantial accretion rates on the structure and evolution of pulsars (see, for example, [1] for a review). In particular, it has become increasingly clear that the magnetic fields $B$ of isolated radio pulsars do not seem to decay on short timescales $\left(\tau_{\text {decay }} \sim 10^{7} \mathrm{yr}\right.$ ) as previously thought; while LMBP show strong evidence for $B$ decay. Accretion has been recently argued [2] to be involved in the very formation of at least one particular system (PSR $1831-00)$ and possibly all the pulsars in these systems by triggering an accretion-induced collapse (AIC) of a progenitor white dwarf; thus avoiding problems with formation in a type II supernova explosion [3].

On the other hand, it has long been speculated that, at least some pulsars (if not all [4-6]) must be strange stars if the strange matter (SQM) conjecture $[4,7]$ is true; in the sense that hadronic matter is a metastable state decaying into a cold $u d s$ plasma (with a net gain of energy) under favorable conditions.

Which exactly are these "favorable conditions" is a matter of some controversy. While the idea of a mixed (neutron + strange) population has been implicit in the literature, there is actually a fine-tunning problem to be explained, namely why a metastable state such as neutron matter can outlive a huge macroscopic time without decaying (we have argued elsewhere that the "natural" conversion is driven by the KelvinHelmholtz timescale $\sim 1 s$ after the formation of a protoneutron star [8]). On the other hand, it has been argued on observational grounds [9] that field radio pulsars can not be identified with strange stars; a strong conclusion that prompted refined calculations of "classical" strange stars [10] and exotic pulsar models [11]. Nevertheless, it should be acknowledged that the widely-spread notion that a neutron star must be highly compressed to reach the neutron $\rightarrow$ strange matter threshold at its center finds a natural setting in binary LMBP where AIC has formed the secondary before the end of the mass-transfer regime.

The purpose of this work is to point out that the identification of pulsars in LMBP systems with strange stars may allow the construction of a consistent scenario for their magnetic, thermal and evolutionary histories. Moreover, the physical features for the model to work seem to emerge quite naturally from simple estimates within reasonable uncertainties.

\section{SCHEMATIC OVERVIEW OF A STRANGE STAR STRUCTURE AND EVOLUTION}

As is well-known, the structure of a strange star $[5,10]$ consists of a dense degenerate SQM core sourrounded by a normal matter crust. Both the Baym-Pethick-Sutherland [12] equation of state (which is generally considered as an accurate description for this density regime) and the case of an accretion-generated crust [13] (which is probably more adequate for the system we are considering) can be parametrized by a polytropic expression $P=K \rho^{\Gamma}$ with sufficient accuracy. For the sake of the argument, we may properly describe the structure of this crust by integrating the hydrostatic equilibrium equation in the approximation $M=$ constant (compare, for example, with [10]) and get

$$
\delta R=\xi \frac{\Gamma}{\Gamma-1} \frac{K R_{s s}^{2}}{G M_{s s}} \rho_{B}^{\Gamma-1}
$$

where $R_{s s}$ is the strange core radius ( $\sim$ star radius for $R_{s s} \gg \delta R$ ), $M_{s s}$ is the mass of the strange star in the same approximation, $\rho_{B}$ is the density at the base of the normal crust and $\xi \sim 0.65$ is a relativistic correction. This approximate (yet accurate) expression for the this crust allows us to relate all the relevant 
quantities to $\rho_{B}$ in a simple and useful way as will be explained below. We shall further assume that the crust forms on short timescales compared to the thermal evolution of the star.

It is widely agreed that, due to the very features of the SQM core, the density of the normal matter at the base of the crust $\rho_{B}$ has to be limited by the neutron drip value $\rho_{D} \simeq 4.3 \times 10^{11} \mathrm{~g} \mathrm{~cm}^{-3}$. However, there is no obvious reason for the latter being the actual value, and any density lower than $\rho_{D}$ is, in principle, possible. In fact, the authors of Ref.[14] have argued that $\sim \rho_{D} / 5$ is the maximum value allowed by considering the mechanical equilibrium of the crust. In the first case, the exact accretion history of the star would be irrelevant, since after the condition $\rho_{B}=\rho_{D}$ is achieved, the dripped neutrons would be swallowed by the SQM core and we will always deal with a maximal crust. On the other hand, if the limiting density is $<\rho_{D}$, the mass of the crust $\delta M\left(\leq 10^{-6} M_{\odot}\right.$ in this approximation) may depend on the history of the object (in the former case, the dominating isotope would be ${ }^{118} \mathrm{Kr}$, while in the latter we would find ${ }^{80} \mathrm{Zn}$ at the base of the crust). Fortunately, since $\Gamma$ is near $4 / 3$, the results will be quite insensitive to the actual value of $\rho_{B}$ provided the crust is not extremely tiny. Keeping this in mind, we shall leave the scaling explicit to a fiducial value $\rho_{0}=10^{11} \mathrm{~g} \mathrm{~cm}^{-3}$ to allow for a range of possibilities. The maximal, neutron drip-limited case is obtained by setting $\rho=4.3 \rho_{0}, Z=36, X=0.3$ below.

The thermal and magnetic history of a strange star will be very different according to the actual state of the SQM core. It has been longly recognized $[15,16]$ that it is entirely possible that the quark liquid forms a superconducting state. The first schematic model calculations of compact stars cooling with superconducting SQM cores [17] have been recently refined and improved [18], to confirm that dramatic effects due to quark pairing can completely alter the quick-cooling signature of strange stars (see, for example, [19]). Since the critical temperature for pairing to occurr is very high in these models $\left(T_{c} \sim 0.1 \mathrm{MeV}\right.$, see $\left.[15,16]\right)$; we can neglect the very short time after the AIC formation event in which the SQM core remains in the normal state. Once it becomes superconducting the magnetic field $B$ should obey the diffusion equation

$$
\nabla^{2} B=\frac{1}{D} \frac{\partial B}{\partial t}
$$

where $D=c^{2} / 4 \pi \sigma_{\text {sqm }}$ is the diffusion coefficient. Acording to Refs.[20], the SQM conductivity $\sigma_{\text {sqm }}$ can be expressed as

$$
\sigma_{s q m} \simeq 10^{19}\left(\alpha_{c} T_{9}\right)^{-5 / 3}\left(\frac{\mu}{300 M e V}\right)^{8 / 3} s^{-1}
$$

where $\alpha_{c}$ is the strong coupling constant, $T_{9} \equiv T / 10^{9} \mathrm{~K}$ is the internal temperature and $\mu$ is the quark chemical potential. The consequence of such a conductivity is immediately clear: unless the estimate (valid for normal SQM) happens to be wrong by many orders of magnitude, the magnetic flux is expulsed in a timescale as short as $\tau_{\text {exp }} \simeq R^{2} / D \simeq 3 \times 10^{3} \mathrm{yr}(\mu / 300 \mathrm{MeV})^{8 / 3}\left(\alpha_{c} T_{9}\right)^{-5 / 3}$; an astronomically small value [21]. Now, the conservation of the expulsed flux demands that the final value of the normal crust grows by a factor $B^{\text {crust }}=B^{\text {core }}(R / \delta R)$ but, because of elastic stresses in the crust can not support magnetic stresses, the $B^{\text {crust }}$ must be limited [22] by $B_{\text {max }}^{\text {crust }}=(8 \pi \mu \Theta \delta R / R)^{1 / 2}$, where $\mu$ is the lattice

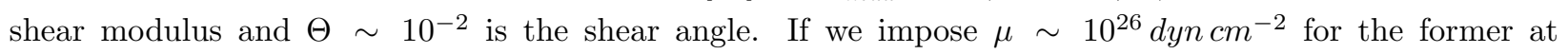
$\rho=\rho_{0}$ then $B_{\text {max }}^{\text {crust }} \simeq 8 \times 10^{11} \mathrm{G}$, which is the initial value expected for the field in a young BP (see below). Because its limited resistance, the crust would be blown off if the initial $B^{\text {core }}$ exceeds the threshold $B_{*}^{\text {core }}=8 \times 10^{11} G(\delta R / R) \sim 10^{10} G$. We suggest that we may in fact be observing those LMBP in which the initial $B^{\text {core }}$ (for which we do not have any reliable information at all) remained lower than $B_{*}^{\text {core }}$. It is remarkable that none of the 24 known systems posesses a $B$ larger than the expected in the model. 


\section{FURTHER EVOLUTION OF THE MAGNETIC FIELD}

Due to ohmic currents the field, now totally confined to the crust if a small skin depth is neglected, should proceed to decay according to

$$
\frac{\partial B}{\partial t}=-\frac{c^{2}}{4 \pi} \nabla \times\left(\frac{1}{\sigma_{\text {crust }}} \nabla \times B\right) .
$$

The solutions which are relevant to our problem are those satisfying $B=0$ immediately above $\rho=\rho_{B}$; in agreement with the complete core flux expulsion expectation. Furthermore, since as discussed above, strange stars with crust satisfy the condition $\delta R / R \ll 1$, we may properly use the "thin crust approximation" $[23]$ to obtain the decay timescale of the longest-lived dipole mode

$$
\tau_{d}=\frac{2 \pi}{c^{2}} \xi \frac{\Gamma}{\Gamma-1} \bar{\sigma}_{\text {crust }} \frac{K R_{s s}^{3}}{G M_{s s}} \rho_{B}^{\Gamma-1} .
$$

where $\bar{\sigma}_{\text {crust }}$ is the average of the electrical conductivity over the crust. Since the conductivity will be dominated by the densest matter [23], an upper limit is set by replacing the average of the conductivity by its value at the drip point (that is $\bar{\sigma}_{\text {crust }} \equiv \sigma_{\text {crust }}\left(\rho=\rho_{B}\right)$ ) hereafter.

As in most field decay models by ohmic dissipation, it is the value of $\sigma_{\text {crust }}$ which controls the behavior of $\tau_{d}$. At the higher temperatures $\sigma_{\text {crust }}$ is dominated by the Umklapp processes [24,25] and reads

$$
\sigma_{U}=1.43 \times 10^{21}\left(\frac{\rho}{\rho_{0}}\right)^{7 / 6}\left(\frac{X}{0.375}\right)^{5 / 3} T_{9}^{-2} s^{-1},
$$

where the fraction of protons per nucleus $X$ has been scaled to its expected value at $\rho_{0}$ and $T_{9} \equiv T / 10^{9} \mathrm{~K}$ identified with the (isothermal) core temperature. Below a freezeout temperature $T_{F}$ the Umklapp processes are frozen and the conductivity is dominated by the impurity concentration $Q$. This $T_{F}$ can be expressed as

$$
T_{F}=1.73 \times 10^{7}\left(\frac{\rho}{\rho_{0}}\right)^{1 / 2}\left(\frac{Z}{30}\right)^{1 / 2}\left(\frac{X}{0.375}\right) K,
$$

where $Z$ is the charge of the dominant isotope ${ }^{80} Z n$ at $\rho_{0}$. Below this temperature the dominant conductivity due to impurity concentration takes the form [25]

$$
\sigma_{I}=4.13 \times 10^{24}\left(\frac{\rho}{\rho_{B}} \frac{X}{0.375}\right)^{1 / 3}\left(\frac{Z}{30}\right)\left(\frac{1}{Q}\right) s^{-1},
$$

where $Q \geq 10^{-3}$ is the mean square deviation of $A$ from its average [26] (it can be readily checked that, unless $Q$ is unexpectedly large, $\sigma_{I}$ does not dominate $\sigma_{U}$ for temperatures $T>T_{F}$ and we shall dismiss this possibility in the remaining of this work).

An important difference between these regimes is that while $\sigma_{U} \propto T^{-2}, \sigma_{I}$ does not depend on the temperature. The crust field decay is therefore different for $T>T_{F}$ than for $T<T_{F}$. In the first case the decay proceeds according to [23]

$$
B^{\text {crust }}(t)=B_{\text {max }}^{\text {crust }} \exp \left(-\int_{0}^{t} \frac{d t^{\prime}}{\tau\left(t^{\prime}\right)}\right)
$$

where 


$$
\tau=7.2 \times 10^{3}\left(\frac{R}{10 k m}\right)^{3}\left(\frac{1.4 M_{\odot}}{M_{s s}}\right)\left(\frac{\rho}{\rho_{0}}\right)^{\Gamma+\frac{1}{6}} \times\left(\frac{X}{0.375}\right)^{3} T_{9}^{-2} y r,
$$

depends on time through the temperature $T$. In the second case, the decay is a simple exponential

$$
B^{\text {crust }}(t)=B_{F}^{\text {crust }} \exp \left(-t / \tau_{I}\right)
$$

with $B_{F}^{\text {crust }}$ the value of the field at the end of the first regime and the time constant is

$$
\tau_{I}=2 \times 10^{7}(R / 10 \mathrm{~km})^{3}(X / 0.375)^{5 / 3} \times\left(\rho / \rho_{0}\right)^{\Gamma-\frac{2}{3}}(Z / 30)(1 / Q) y r,
$$

which happens to be always $\geq 10^{9} \mathrm{yr}$ for the expected parameters unless $Q$ happens to be very high.

A determination of the evolution of $B^{\text {crust }}(t)$ for $T>T_{F}$ requires the knowledge of the thermal history $T(t)$. The important point here is that SQM superconductivity renders a plateau in the $T$ vs. age curve $[17,18]$ (which is absent in the case of a normal SQM core). The boldest approximation is to set $T=$ constant for the plateau era and use the relationship between the surface temperature and core temperature given in Ref.[27]; namely $T_{s} \simeq 10^{6}\left(T / 10^{8} K\right)^{0.55} K$ (which has been argued to be valid for strange stars as well [28]) to yield the crust field at any time $t \leq t_{p l}$

$$
B^{\text {crust }}(t)=B_{\text {max }}^{\text {crust }} \exp \left[-\frac{1}{7.2}\left(\frac{t}{10^{5} y r}\right)\left(\frac{<T_{s}>}{10^{6} K}\right)^{3.64}\right]
$$

where $\left\langle T_{s}\right\rangle$ is the average of the surface temperature in the plateau era. Since, according to recent calculations [18], $<T_{s}>\simeq 2 \times 10^{6} \mathrm{~K}$, we conclude that the field should decay by a factor of $\geq 10^{2}$ along the plateau era lasting $f e w \times 10^{5} \mathrm{yr}$. Using a more accurate fit to Ref.[18] results $T_{s}=10^{6.5}(\mathrm{yr} / \mathrm{t})^{0.05} \mathrm{~K}$ we find the refined estimate of the decay along this epoch

$$
B_{F}^{\text {crust }}=B_{\text {max }}^{\text {crust }} \exp \left[-1.45\left(\frac{t}{10^{5} y r}\right)^{0.82}\right]
$$

which leads to essentially identical conclusions.

\section{DISCUSSION}

Based on the results of the former section, it is tempting to suggest that a decay of the field by a factor of $\sim 10^{3}$ in the first $10^{5}-10^{6} \mathrm{yr}$ is built-in by the physics of strange star crusts with superconducting SQM cores. The same line of reasoning shows that, unless the impurity concentration is very high $\left(Q>10^{-2}\right)$, further decay of $B^{\text {crust }}$ below $B_{F}^{\text {crust }} \sim 10^{9} G$ is inhibited and its value remains effectively frozen because of the larger value of the decay constant $\geq 10^{9} \mathrm{yr}$, again an effect controlled by the microphysics of the thin normal crust of a strange star.

From the astrophysical point of view a model in which LMBPs form from a symbiotic system $\left(\sim 1 M_{\odot}\right.$ low-mass giant + white dwarf) is attractive since the latter are abundant in the galaxy. According to the accepted scenario (see for example [29]), as the non-degenerate star leaves the main sequence its radius increases until filling the critical lobe and mass transfer starts. Along this mass-transfer stage AIC of the white dwarf happens, and because of the $\sim 0.1 M_{\odot}$ energy loss, the binary temporarily detaches. When accretion resumes the collapse of the neutron star to a strange star should follow after the accretion of $\geq 0.1 M_{\odot}$ from the companion, which is sufficient to drive the conversion by compression. The SS is born 
hot but must cool very quickly below $T_{c}$, and therefore expulse the interior field as described (the latest work on quark pairing seems to suggest much larger gaps of $\sim 100 \mathrm{MeV}$ with potential important effects on the cooling which have not been explored as yet, see for example [30]). After $\sim$ few $\times 10^{5} \mathrm{yr}$ this field would decay to the "bottom value" $B_{F}^{\text {crust }} \simeq 10^{8} G$ (eqs. 9-11). We suggest that systems like PSR 1718-19 and PSR 1831-00 are quite young and their evolution downwards in the $B_{s}-P_{\text {orb }}$ plane (Fig. 1 of Ref. [2]) may be measurable. It is also important to note that spin periods in the millisecond range are possible for strange stars in LMBP [31], in agreement with observations; while they would be prohibited in the case of a neutron composition because of r-mode instability [32].

Our estimations above may be helpful for an interpretation of why we should expect the field to decay differently on two different timescales, a point not easily made for neutron star models where the much denser crust behaves differently and complete flux expulsion remains controversial. We finally remark that, at least in principle, the identification is also consistent with the lack of glitches and substantial timing noise

of pulsars in LMBP systems (which is naturally expected from strange stars [33]); and explains the sharp contrast with isolated field radio pulsars $B$ evolution, whose composition need not to be exotic because of formation arguments.

\section{ACKNOWLEDGEMENTS}

We would like to acknowledge the financial support of the Brazilian Agencies FAPESP (São Paulo) and CNPq through several forms of grants. M.P.Allen, P.Benaglia, G.A.Romero and, particularly, J.A.de Freitas Pacheco are greatfully acknowledged for useful suggestions.

\section{REFERENCES}

[1] D.Bhattacharya and E.P.J.van den Heuvel, Phys.Rep. 203, 1 (1991).

[2] E.P.J.van den Heuvel and O.Bitzaraki, Astron.Astrophys. 297, L41 (1995).

[3] D.J.Helfand, M.Ruderman and J.Shaham, Nature 304, 423 (1983).

[4] E.Witten, Phys. Rev. D 30, 272 (1984).

[5] C.Alcock, E.Farhi and A.V.Olinto, Astrophys.J. 310, 261 (1986) ; P.Haensel, J.Zudnik and R. Schaeffer, Astron. Astrophys. 160, 121 (1986).

[6] O.G.Benvenuto, J.E.Horvath and H.Vucetich, Int.Jour.Mod.Phys. A 6, 4769 (1991).

[7] A.Bodmer, Phys. Rev. D 4, 1601 (1971), see also H. Terazawa INS Report 338 (INS, Univ. of Tokyo, 1979) and S. A. Chin and A.K. Kerman, Phys. Rev. Lett. 43, 1292 (1979).

[8] O.G.Benvenuto and J.E.Horvath, Phys. Rev. Lett. 63, 716 (1989).

[9] M.A.Alpar, Phys. Rev. Lett. 58, 2152 (1987).

[10] N.K.Glendenning and F.Weber, Astrophys.J. 400, 647 (1992).

[11] O.G.Benvenuto, J.E.Horvath and H.Vucetich, Phys. Rev. Lett. 64, 713 (1990).

[12] G.Baym, C.Pethick and P.Sutherland, Astrophys.J. 170, 299 (1971).

[13] P. Haensel and J.L. Zdunik, Astron. Astrophys. 229, 117 (1990).

[14] Y.F.Huang and T.Lu, Astron.Astrophys.325, 189 (1997).

[15] D.Bailin and A.Love, Phys. Rep. 107, 325 (1984).

[16] J.E.Horvath, O.G.Benvenuto and H.Vucetich, Mod. Phys. Lett. A 7, 995 (1992).

[17] J.E.Horvath, O.G.Benvenuto and H.Vucetich, Phys. Rev. D 44, 1147 (1991).

[18] C.Schaab, B.Hermann, F.Weber and M.K.Wiegel, Astrophys.J.Lett. 480, 111 (1997).

[19] P.Pizzochero, Phys. Rev. Lett. 66, 2425 (1991). 
[20] H. Heiselberg and C.J. Pethick, Phys. Rev. D 48, 2916 (1993), note that this refined calculations give a different behavior than, e.g. P.Haensel and A.J.Jerzak, Acta Phys. Pol. B 20, 141 (1989).

[21] Bailin and Love op. cit. had in fact first speculated that very short expulsion timescales were possible. [22] M.Ruderman, Astrophys.J. 382, 576 (1991).

[23] C.Pethick and M.Sharling, Astrophys.J. 453, L29 (1995).

[24] V.A.Urpin, Sov. Astron. 36, 393 (1992).

[25] V.A.Urpin and D.G.Yakovlev, Sov. Astron. 24, 303 (1980).

[26] E.Flowers and M.A.Ruderman, Astrophys.J. 215, 302 (1977).

[27] E.H.Gudmunsson, C.J.Pethick and R.Epstein, Astrophys.J. 272, 286 (1983).

[28] V.V.Usov, Astrophys.J.Lett. 481, L107 (1997).

[29] M. de Kool and J. van Paradijs, Astron.Astrophys. 173, 279 (1987).

[30] T. Schaefer and F. Wilczek, hep-ph/9906512

[31] J. Madsen, Phys. Rev. Lett. 81, 3311 (1998).

[32] N. Andersson, K. Kokkotas and B.F. Schutz, Astrophys. J 510, 846 (1999).

[33] P.B. Jones, Mon. Not. R.A.S. 246, 364 (1990). 